\title{
Enfermedad de Chagas-Mazza congenita en Salta
}

\author{
Congenital Chagas-Mazza disease in Salta, Argentina \\ Silvia Contreras, María Rosa Fernandez, Fernando Agüero, \\ Javier Desse Desse, Tomás Orduna y Olindo Martino
}

\begin{abstract}
Resumen Se estudió la infección por T. cruzi en mujeres embarazadas en la localidad de General Güemes, provincia de Salta. La misma fue del 12,3\%. El 8,8\% de los recién nacidos estudiados tuvieron diagnóstico de Enfermedad de Chagas utilizando la técnica directa (microhematocrito). Todos fueron tratados con benznidazol a razón de $5 \mathrm{mg} / \mathrm{kg} /$ día durante 30 dias. Todos presentaron anemia, que fue interpretada como reacción adversa medicamentosa. Se estima que la técnica directa representa la mejor opción para llevar a cabo el diagnóstico de esta enfermedad en el recién nacido. Se ha propuesto un flujograma para el seguimiento de la infección por T. cruzi en el recién nacido.
\end{abstract}

Palabras-claves: Enfermedad de Chagas-Mazza. Transmisión congénita. Incidencia. Diagnóstico. Tratamiento.

\begin{abstract}
A study was carried out on T. cruzi infection in pregnant women in the Guemes area in Salta province; the rate of infection was $12.3 \%$. Of newborn children studied by the microhematocrit technique, $8.8 \%$ were diagnosed with Chagas' disease. All of them were treated with benznidazol $(5 \mathrm{mg} / \mathrm{kg} /$ day) for one month; all of them presented anemia, which was interpreted to be an adverse drug reaction. The microhematocrit technique is believed to represent the best option for diagnosis of this disease in newborn children. A diagnostic methodology to follow the course of the infection is proposed.
\end{abstract}

Key-words: Chagas-Mazza disease. Congenital transmission. Incidence. Diagnosis. Treatment.

La enfermedad de Chagas-Mazza o trypanosomiasis americana, es una zoonosis parasitaria hística y hemática cuyo agente etiológico es Trypanosoma cruzi (T. cruzi), protozoario flagelado hematófilo que anida y se reproduce en tejidos (familia Trypanosomatidae) $)^{41}$. Representa una patología endémica rural limitada al continente americano $^{34}$, que se extiende entre el paralelo $42^{\circ}$ Latitud Norte y el paralelo $45^{\circ}$ Latitud Sur.

Es causa de infección entre 16 y 18 millones de personas ${ }^{45}$ y provoca la muerte de 50.000 personas por año ${ }^{16}$.
La infección guarda estrecha relación con la ignorancia, la pobreza, la falta de higiene y la inercia ${ }^{34}$. Se describen tres vías principales de infección: la vectorial, a través de insectos vectores hematófagos triatomineos; la transfusional, principal fuente fuera del área endémica ${ }^{39} 43$ y la via transplacentaria (o congénita). En esta comunicación se describen los aspectos epidemiológicos, clínicos, diagnósticos y terapéuticos de la infección congénita por T. cruzi detectados en la ciudad de General Güemes , Provincia de Salta (Argentina).

Servicio de Neonatología, Hospital "Dr. Joaquín Castellanos,. General Güemes, Salta, y Comisión Nacional para el Estudio de la Patología Regional Argentina (CEPRA), Hospital de Enfermedades Infecciosas "Dr. Francisco Javier Muñiz". Buenos Aires, Argentina. Dirección para correspondencia: Prof. Olindo Martino. Hospital de Enfermedades Infecciosas "Dr. Francisco Javier Muñiz". (CEMPRA-MT). Uspallata 2272, Capital Federal. (1282). República Argentina

Fax: 54-11-4304-2386

E-mail: cempramt@intramed.net.ar

Recebido para publicação em 23/8/98. 


\section{MATERIAL Y MÉTODOS}

Se estudiaron 276 embarazadas durante el período comprendido entre agosto y diciembre de 1996. Se investigó la infección por T. cruzien todas ellas, $y$ del parásito en los niños recién nacidos de las madres infectadas.

El diagnóstico de infección por $T$. cruzi en mujeres embarazadas se basó en la presencia de serología positiva en dos oportunidades realizadas con diferentes técnicas. Para ello se utilizaron la hemaglutinación ${ }^{15}$ y la técnica de ELISA $^{21}{ }^{46}$. En los recién nacidos, el criterio utilizado fue la positividad de una técnica parasitológica directa (microhematocrito).

Todos los recién nacidos con infección por T. cruzi fueron tratados con benznidazol, a razón de $5 \mathrm{mg} / \mathrm{kg} / \mathrm{dia}$, durante 30 días.

\section{RESULTADOS}

Se determinó que la prevalencia de la infección entre la población de mujeres embarazadas fue del 12,3\% (34/276). De los 34 hijos de madres chagásicas, el 8,8\% (3/34) tuvieron infección activa. De los niños estudiados se encontró que el $100 \%$ eran recién nacidos de término, con peso adecuado para la edad gestacional, asintomáticos y con valores de laboratorio dentro de límites normales.
Todos los niños negativizaron el microhematocrito tras quince días de terapéutica, mientras que dos de ellos negativizaron su serología a los cuatro meses.

Todos presentaron anemia, interpretada como reacción adversa al medicamento instituído. Uno de ellos acusó irritabilidad.

\section{DISCUSIÓN}

La prevalencia de mujeres infectadas con T. cruzi varía de acuerdo a la región geográfica y a las características socioeconómicas de los grupos estudiados. En la Argentina se sitúa entre el $4 \%{ }^{3}$ y el $52 \%{ }^{20}$, según la zona estudiada. Nuestro estudio reveló una prevalencia del 12,3\%, similar a la encontrada por otro grupo de trabajo en la misma zona. La mayoría de las mujeres embarazadas no presentaron signos ni síntomas atribuíbles a la infección chagásica ${ }^{3}$.

Durante el embarazo las mujeres infectadas en fase aguda ó crónica pueden transmitir T. cruzi a través de la placenta ${ }^{19}$. La infección fetal se produce como consecuencia de la presencia de parasitemia materna ${ }^{28} 31$. La transmisión congénita de $T$. cruzi oscila entre $0,7 \%$ y el $10 \%$, según los diversos autores 23253738 . En nuestro relevamiento se registró $8,8 \%$ de infección congénita, lo que duplica los valores históricos reportados en esta región del país ${ }^{48}$. El parásito puede comprometer al feto $\mathrm{y} / 0$ a la placenta ${ }^{5632}$.

Luego de la infección el feto puede sufrir alteraciones en su viabilidad y/o en su crecimiento, de acuerdo al momento en que suceda la parasitación. La Enfermedad de Chagas-Mazza congénita puede producir aborto espontáneo ${ }^{35}$, nacimiento prematuro ${ }^{2236}$, retardo de crecimiento y mortinatos ${ }^{724}$.

El espectro clínico de la infección congénita es amplio y puede abarcar desde formas graves $^{79243647}$ hasta formas asintomáticas ${ }^{3947}$.
Los recién nacidos chagásicos estudiados en el presente trabajo fueron asintomáticos.

En cuanto al método diagnóstico en los recién nacidos se considera a la técnica directa de elección (microhematocrito), ya que permite efectuar un diagnóstico rápido con una sensibilidad del 97,4\% ${ }^{17} 1931$.

En los niños mayores de 6 meses adquieren importancia estudios serológicos pués en este grupo de pacientes es donde el microhematocrito disminuye su sensibilidad como consecuencia de la decreciente carga parasitaria circulante ${ }^{19} \mathrm{y}$, por otra parte, desaparecen los anticuerpos tipo lgG transmitidos pasivamente por la madre a través de la placenta ${ }^{27}$. El criterio utilizado para determinar si existe infección en estos pacientes es la reactividad para dos pruebas serológicas realizadas con técnicas diferentes ${ }^{4} 141942$. El diagnóstico precoz permite instaurar el tratamiento parasiticida el cual es más efectivo si se inicia tempranamente ${ }^{12}$.

Las drogas de elección para el tratamiento de la Enfermedad de Chagas-Mazza congénita son nifurtimox ${ }^{243035} \mathrm{y}$ benznidazol ${ }^{126}$. Este última se usa en dosis de $5-7 \mathrm{mg} / \mathrm{kg} /$ dia durante 30 dias. La respuesta terapéutica es evaluada con microhematocrito en los recién nacidos el cual suele negativizarse en el $100 \%$ de los casos a las tres semanas ${ }^{19}$, hecho corroborado en nuestro estudio. Los efectos adversos más frecuentes son exantema, polineuropatía 
periférica, depresión de la médula ósea y síntomas gastrointestinales ${ }^{34}$. Los linfomas descriptos en conejos ${ }^{43}$ no ocurren en el hombre ${ }^{34}$. Los pacientes estudiados presentaron anemia en el $100 \%$ de los casos y uno de ellos acusó irritabilidad.

Dada la relevancia que adquiere la endemia chagásica en nuestro continente, revelado en esta ocasión por la alta incidencia de niños chagásicos en tan sólo seis meses de estudio en una provincia de nuestro país, creemos necesaria la implementación de todas aquellas medidas que tienden a identificar a todas las mujeres embarazadas con infección por T. cruzi. Es por ello que consideramos apropiado el control serológico de la totalidad de las mujeres embarazadas y proponemos el tratamiento con fármacos parasiticidas a todo niño mayor de 6 meses con 2 pruebas serológicas positivas.

\section{REFERENCIAS BIBLIOGRÁFICAS}

1. Andrade S, Magalhaes J, Pontes A. Evaluation of chemotherapy with benznidazole and nifurtimox in mice infected with Trypanosoma cruzistrains of different types. Bulletin of the World Health Organization 63:721-726, 1985.

2. Azogue E, La Fuente C, Darras C. Congenital Chagas' disease in Bolivia: epidemiological aspects and pathological findings. Transactions of the Royal Society of Tropical Medicine and Hygiene 79:176-180, 1985.

3. Barousse A, Esposto M, Mandel S. Evolución serológica de recién nacidos de madres chagásicas en área no endémica. Medicina (Buenos Aires) 35:436-437, 1975.

4. Barousse A, Esposto M, Mandel S., Sousa Martinez F. Enfermedad de Chagas congénita en area no endémica. Medicina (Buenos Aires) 38:611-615,1978.

5. Bittencourt $A$. Doença de Chagas congénita na Bahia Revista Baiana de Saúde Pública 11:159-209, 1984.

6. Bittencourt A, Barbosa H. Incidencia da transmissâo congénita da doenca de Chagas em abortos. Revista do Instituto de MedicinaTropical de Sâo Paulo 14: 257-259, 1972.

7. BittencourA, Barbosa H, Santos $Y$, Ramos M. Incidencia da transmissâo congénita da doenca de Chagas en partos a termo. Revista do Instituto de Medicina Tropical de São Paulo 16:197-201, 1974.

8. Bittencourt A, Mota E, Ribeiro R. Incidence of congenital Chagas' disease in Bahia, Brazil. Journal of Tropical Pediatrics 31:242-248,1985.

9. Bittencourt A, Sandigursky M, Barbosa H. Doenca de Chagas congénita. Estudio de 29 casos. Revista do Instituto de Medicina Tropical de Sâo Paulo 17:146-159, 1975.

10. Blank B. Enfermedad de Chagas y embarazo. Estado actual del tema. Revista de la Sociedad Argentina de Ginecología y Obstetricia (Buenos Aires) 53:311-315, 1974.

11. Blank B, Bonet A, Cichero J, Ortiz S, Del boca J, Villagra L, Mosquera J, Paolasso E. La infección chagásica fetoneonatal en relación con la infeción chagásica materna. Revista de la Sociedad Argentina de Ginecología y Obstetricia (Buenos Aires) 50:324-326, 1971.
12. Brener Z. Present status of chemotherapy and chemoprophylaxis of human trypanosomiasis in the western hemisphere. Pharmacology Therapeutics 7:7190, 1979

13. Camargo M, Taleda G. Diagnóstico de laboratorio. In: Brener Z, Andrade Z (eds) Trypanosoma cruzie doença de Chagas, Guanabara Koogan, Rio de Janeiro, 1979.

14. Cerisola J, Fatala Chaben M, Lazari J. Test de Hemaoaglutinación para el diagnóstico de la enfermedad de Chagas. La Prensa Médica Argentina 49:1761-1765, 1967.

15. Espinosa R, Carrasco H, Belandria F. Life expectancy analysis in patients with Chagas' disease: prognosis after one decade (1973-1983). Internal Journal of Cardiology 8:48-56, 1985.

16. Freilij $\mathrm{H}$, Altcheh J. Chagas congénito. In: Storino R, Milei, $\mathrm{J}$ (eds) Enfermedad de Chagas, 1ra edición, Doyma, Buenos Aires, p. 267-278, 1994.

17. Freilij H, Altcheh J. Congenital Chagas' disease: diagnostic and clinical aspects. Clinical Infectious Diseases 21:551-555, 1995.

18. Freilij H, Muller L, González Cappa S. Direct micromethod for diagnosis of acute and congenital Chagas' disease. Journal of Clinical Microbiology 18:327-330, 1983.

19. Guillerón C, Gorodner J. Características de la infección chagásica en gestantes y recién nacidos de la maternidad Castelli, Chaco. Argentina. I Congreso Brasileiro de Infectologia, 1982.

20. Hermitte G, Moreno M, Pérez A, Segura E. Títulos serológicos y estado clínico del paciente chagásico de evolución tardía. Congreso Argentino de Protozoología y Reunión sobre Enfermedad de Chagas, Huerta Grande, Córdoba, 1984.

21. Howard J. Clinical aspects of congenital Chagas' disease. American Tryponosomiasis Research. Pan American Health Organization/WHO. Scientific Publication. no 318 . World Health Organization, Geneva. p. 212-221, 1975

22. Howard J, Rios C, Espensperger I, Olivos P. Enfermedad de Chagas congénita. Boletín Chileno de Parasitología 14:42-45, 1957.

23. Howard JE. La enfermedad de Chagas congénita. Tesis de Doctorado. Universidad de Chile, Santiago, Chile,1962. 
24. Lisboa Bittencourt A. Congenital Chagas' disease. American Journal Diseases of Children 130:97-103, 1976.

25. Lopes ER, Chapadeiro E, Rodriguez da Cunha M, Fonseca M. Doença de Chagas congénita no Triángulo Mineiro. Relato de um caso. Revista do Instituto de Medicina Tropical de Sâo Paulo 19:165-169, 1977.

26. Marr J, Docampo R. Chemotherapy for Chagas' disease: a perspective of current therapy and considerations for future research. Reviews Infectious Diseases 8:884-903, 1986.

27. Miles M. Trypanosoma cruzi milk transmission of infection and immunity from mother to young. Parasitology $65: 1$ 9, 1972.

28. Moya P. El hijo de madre chagásica.Tesis de Doctorado. Facultad de Ciencias Médicas. Universidad Nacional de Córdoba, 1977.

29. Mota P. Enfermedad de Chagas congénita: experiencia clínica. In: Congreso Argentino de Protozoología y Reunión sobre Enfermedad de Chagas. Huerta Grande. Córdoba, 1984.

30. Moya P, Moretti E, Paolasso R. Enfermedad de Chagas neonatal. Diagnóstico de laboratorio en el primer año de vida. Medicina (Buenos Aires) 49:595-599, 1989.

31. Moya P, Paolasso R, Blanco S. Tratamiento de la Enfermedad de Chagas con nifurtimox durante los primeros meses de vida. Medicina (Buenos Aires) 45:553558, 1985.

32. Moya P, Villagra L, Risco J. Enfermedad de Chagas congénita: hallazgos anatomopatológicos en placenta y cordón umbilical. Revista de la Facultad de Ciencias Medicas de la Universidad de Córdoba 37:21-27, 1979.

33. Muñoz P, Thiermann E, Atías M, Acevedo C. Enfermedad de Chagas sintomática en recién nacidos y lactantes. Boletín Chileno de Parasitología 47:35-39, 1992.

34. Prata A. Enfermedad de Chagas. In: Enfermedades latinoamericanas. Clínicas de Infectología de Norteamérica. Editorial Intermédica, BuenosAires, p. 6178, 1995.

35. Saleme A, Yanicelli G, Iñigo L. Enfermedad de ChagasMazza en Tucumán. Archivos Argentinos de Pediatría 69:162-169, 1971.

36. Saleme A, Yanicelli L, Iñigo L, Moreno A, Valperg S, Alonso E, Paz A, García Toro A, Graña E. Enfermedad de Chagas-Mazza congénita. In:XXI Jornadas Argentinas de Pediatría. Rio Hondo, 1971

37. Schmuñis G. Chagas' disease and blood transfusion. In: Dood R, Barker L (eds) Infection, inmunity and blood transfusion. New York, 1984.

38. Schmuñis G, Szarfman A. La enfermedad de Chagas congénita. Medicina (Buenos Aires) 37:47-53, 1977.

39. Storino R. Evolución natural y fisiopatogenia de la Enfermedad de Chagas humana. Tesis de Doctorado. Universidad Nacional de La Plata. Facultad de Ciencias Médicas, 1984.

40. Storino R, Milei J. Miocardiopatía chagásica crónica, un enfoque para el clinico general. Ed. Club de Estudio. Buenos Aires, Argentina, 1986.

41. Szarfman A, Urman J, Otalora A, Larguía A, Yanovsky J. Specific agglutinins and immunoglobulin levels in congenital Chagas' infections. Medicina (Buenos Aires) 35:245-250, 1975.

42. Texeira A, Cordoba J, Msior Y. Chagas' disease lymphoma growth in rabbits treated with Benznidazole. American Journal of Tropical Medicine and Hygiene 43:146-158, 1990.

43. Tonn R. Review of recent publication on the ecology, biology and control vectors of Chagas' disease. Revista Argentina de Microbiología 20:4-11, 1986.

44. Voller A, Draper C, Bidwell D, Aertlett A. A microplate enzime linked immunoabsorbent assay for Chagas' disease. Lancet 1(7904):426-428, 1975.

45. Votta R, Marchese C, Sousa Martinez F. La Enfermedad de Chagas en la embarazada y el recién nacido. Revista de la Sociedad Argentina de Ginecología y Obstetricia 53:56-58, 1974.

46. World Health Organization. Special Programme for Research and Training in Tropical Diseases. Tenth programme report. Chpter 7. Chagas's disease. World Health Organization, 1991.

47. Zaidemberg M, Segovia A. Enfermedad de Chagas congénita en la ciudad de Salta, Argentina. Revista do Instituto de Medicina Tropical de Sâo Paulo 35:35-43, 1993. 